\title{
Analysis of the variability of the luminous emission line star MWC 314
}

\author{
G. Muratorio ${ }^{1}$, C. Rossi $^{2}$, and M. Friedjung ${ }^{3}$ \\ 1 Laboratoire d'Astrophysique de Marseille, 2 Place le Verrier, 13248 Marseille Cedex 04, France \\ e-mail: gerard.muratorio@oamp.fr \\ 2 Dipartimento di Fisica, Università "La Sapienza", Piazzzale A. Moro 2, 00185 Roma, Italy \\ e-mail: corinne.rossi@roma1.infn.it \\ 3 Institut d'Astrophysique, 98 bis Boulevard Arago, 75014 Paris, France \\ e-mail: fried@iap.fr
}

Received 27 October 2007 / Accepted 20 May 2008

ABSTRACT

\begin{abstract}
Context. We investigated the surroundings of MWC 314 in the framework of the study of hot emission line star environments using the SAC method. This star is either a B[e] supergiant or a luminous blue variable and appears to be extremely luminous and massive. Aims. We determine the structure and physical conditions of the emitting region and study the possible variations.

Methods. We measured the absorption and emission line radial velocities and the emission line fluxes on high-resolution spectra obtained with Aurelie at the $1.52 \mathrm{~m}$ OHP telescope in July 1998, with Elodie at the $1.93 \mathrm{~m}$ OHP telescope at various epochs, and with echelle spectrographs of the Asiago and Loiano observatories (Italy) in 2006. We used the statistical approach of the self-absorption curve method (SAC) to derive physical parameters of the line-emitting region.

Results. We detected drastic variations of the photospheric absorption line radial velocities with time, while the emission line velocities appear to be stable. The Cr II, Ti II, and Fe II emission lines have a complex structure. They are double-peaked, and each of these two $60 \mathrm{~km} \mathrm{~s}^{-1}$ separated components, is composed of a narrow and a broad component, while the [Fe II] line components are narrower. The fit of the various components of the Fe II lines to a SAC curve indicates that their intensities are affected by some self absorption. We obtained a Boltzmann-type population law whose mean excitation temperature is $6500_{-1000}^{+1500} \mathrm{~K}$ for the narrow component lower and upper levels. We obtained a higher Boltzmann-type population law of $10500_{-2000}^{+3000} \mathrm{~K}$ for the forbidden transition upper levels. Conclusions. From the absorption lines we confirm the binarity for MWC 314. The periodicity has nevertheless to be improved with a higher sampling frequency. Our results from the emission lines are consistent with line formation in a rotating disk around a star. The typical minimum radius of the line emitting region obtained from the SAC study is $3.5 \times 10^{13} \mathrm{~cm}\left(2.0 \times 10^{13} \mathrm{~cm}<R<6.3 \times\right.$ $10^{13} \mathrm{~cm}$ ). We argue, in the framework of a very simplified geometrical model, that the [Fe II] lines are emitted farther out than the permitted $\mathrm{Cr}$ II, Ti II, and Fe II lines, in a disk inclined $25 \pm 5$ degrees to the plane of sky. If the rotation of the disk is Keplerian, the Fe II lines are emitted in a zone defined by $4 \times 10^{12} \mathrm{~cm}<R<7 \times 10^{13} \mathrm{~cm}$, while for a rotation with conservation of angular momentum, they are emitted from $4 \times 10^{12} \mathrm{~cm}<R<2 \times 10^{13} \mathrm{~cm}$.
\end{abstract}

Key words. line: formation - methods: data analysis - stars: emission-line, Be - stars: early-type

\section{Introduction}

MWC $314=\mathrm{BD}+14^{\circ} 3887$ has been classified as a candidate LBV by Miroshnichenko et al. (1998) and, more recently, by Wisniewski et al. (2006), but its spectral characteristics do not rule out the possibility that it is classified as a $\mathrm{B}[\mathrm{e}]$-supergiant (Zickgraf et al. 1986). Both these classes appear to be evolved, very massive stars, which have not been understood very well. This is particularly true for their strong winds, including the slow disk confined winds of $\mathrm{B}[\mathrm{e}]$ supergiants. Ever since Merill (1927) first examined this star, it has not been observed much compared with similar objects. Swensson (1942) identified numerous emissions of metallic lines including those of Fe II, [Fe II] Cr II, and Ti II.

Miroshnichenko (1996) offers an exhaustive bibliography. The SED of this object can, according to him, be compared alternatively with P Cygni (with a higher reddening) or to XX Oph

* based on observations obtained at the Loiano and Asiago Observatories and with AURELIE spectra and ELODIE archival spectra of the Haute Provence Observatory. (a B0-M binary). He reports relatively small photometric variations with an amplitude of 0.3 in all bands (UBVRIJHK). Miroshnichenko et al. (1998) confirm that MWC 314 is one of the most luminous stars in the Milky Way from its parameters. They observed photospheric lines for the first time.

Wisniewski et al. (2006) report the results of a spectroscopic and spectropolarimetric monitoring between 1999 and 2004. Their observations "exhibit substantial variability on timescales of several days to weeks" both in the equivalent width of $\mathrm{H} \alpha$ and in the $R$-band polarization. They find evidence of an asymmetrical circumstellar envelope.

Very recently, Marston \& McCollum (2008) from H $\alpha$ narrow band images have found a large bipolar nebula associated with MWC 314. It shows no evidence of processed materials. The timescale for the formation of such a structure should be more than $10^{5}$ years at the upper end for LBV nebular expansion. These authors conclude that there is no clear evidence that nebula was formed during an LBV stage of the star.

We performed our first observations of MWC 314 in the framework of our study of hot emission-line star environments. 
Table 1. Log of the spectroscopic observations of MWC 314.

\begin{tabular}{ccccccc}
\hline \hline Date & UT & Exp & Sp Range & $R$ & $S / N$ & Inst \\
\hline $28 / 8 / 94$ & $21: 30$ & 2877 & $3850-6800$ & 42000 & 40 & 2 \\
$7 / 6 / 95$ & $24: 45$ & 5400 & $3850-6800$ & 42000 & 89 & 2 \\
$19 / 8 / 95$ & $23: 07$ & 3600 & $3850-6800$ & 42000 & 63 & 2 \\
$2 / 7 / 98$ & $23: 00$ & 5000 & $4050-4490$ & 7000 & 10 & 1 \\
$3 / 7 / 98$ & $23: 40$ & 7200 & $4485-4915$ & 7000 & 20 & 1 \\
$5 / 7 / 98$ & $23: 30$ & 7200 & $5315-5745$ & 10000 & 100 & 1 \\
$7 / 7 / 98$ & $22: 30$ & 5500 & $5750-6600$ & 5000 & 100 & 1 \\
$8 / 7 / 98$ & $22: 20$ & 10800 & $4900-5320$ & 8000 & 120 & 1 \\
$9 / 7 / 98$ & $22: 30$ & 7200 & $6145-6355$ & 20000 & 80 & 1 \\
$21 / 7 / 98$ & $23: 40$ & 4700 & $5325-5535$ & 19000 & 80 & 1 \\
$22 / 7 / 98$ & $22: 00$ & 9000 & $5120-5330$ & 18000 & 100 & 1 \\
$9 / 9 / 98$ & $19: 50$ & 3600 & $3850-6800$ & 42000 & 78 & 2 \\
$26 / 6 / 06$ & $23: 30$ & 1800 & $3900-7000$ & 5000 & 200 & 3 \\
$16 / 7 / 06$ & $21: 40$ & 1800 & $4300-7000$ & 20000 & 100 & 4 \\
\hline
\end{tabular}

Starting from the evidence of a circumstellar envelope characterized by numerous emission lines we found this object to be a good target for study by means of the SAC method to determine the structure and the physical conditions of the emitting regions.

Subsequently the variability detected by Wisniewski et al. (2006) and our serendipitous finding of radial velocity variability of the photospheric absorption lines further stimulated our interest in the study of this object whose nature still seems to be poorly understood (Marston \& McCollum 2008).

\section{Observations and reductions}

We observed MWC 314 at the Haute Provence Observatory during two periods with AURELIE in July 1998. We completed these observations with four spectra that are available in the ELODIE archive and with a spectrum obtained in June 2006 at the Loiano observatory and one in July 2006 at the Asiago observatory. A detailed log of the observations is given in Table 1. UT is the mid-exposure time. The exposure time is given in seconds and spectral range in $\AA$. $S / N$ ratio refers to the continuum. In the column Inst: 1 is AURELIE, 2 is ELODIE, 3 is LOIANO, 4 is ASIAGO. The radial velocity stability of the various spectrographs is confirmed by the stability of the emission line radial velocity with time.

The spectra were reduced at the Marseille Observatory using procedures running in the MIDAS environment. The files were normalized to the continuum. The flux values were then multiplied by the SED derived from a spectrum obtained at the LOIANO observatory in 2006 at low resolution. This file was dereddenned with $E(B-V)=1.84$ from Miroshnichenko (1996). Although Wisniewski et al. (2006) detected the first conclusive evidence of $\mathrm{H} \alpha$ equivalent width variability, Miroshnichenko et al. (1998) claim that their observations show no significant variations in the line intensities or profiles, which enables us to adopt for all our data the SED derived in 2006.

\section{Analysis of the variations of the photospheric lines}

Table 2 gives the mean heliocentric velocities of the few absorption lines observed in the spectra according to the list given by Miroshnichenko et al. (1998). The first column refers to the points in Fig. 9. The third column gives the Julian date $(+24400000)$. The fourth column gives the $\mathrm{He} \mathrm{I}$ and $\mathrm{Mg}$ II radial velocity, standard deviation of the line set in $\mathrm{km} \mathrm{s}^{-1}$,
Table 2. Mean absorption component radial velocities for the various epochs of observation.

\begin{tabular}{cccccc}
\hline \hline$N$ & Date & JD & HeI, MgII & Phot. & Phase \\
\hline 1 & $28 / 8 / 1994$ & 9593 & $-16 \pm 4(7)$ & $-25 \pm 7(10)$ & 0.65 \\
2 & $7 / 6 / 1995$ & 9876 & $96 \pm 11(7)$ & $95 \pm 3(17)$ & 0.86 \\
3 & $19 / 8 / 1995$ & 9949 & $42 \pm 3(4)$ & $47 \pm 5(16)$ & 0.23 \\
4 & $2-9 / 7 / 1998$ & 10998 & $-62 \pm 2(3)$ & $-80 \pm 6(10)$ & 0.43 \\
5 & $21-22 / 7 / 1998$ & 11016 & $\ldots \ldots \ldots$ & $99 \pm 13(6)$ & 0.98 \\
6 & $9 / 9 / 1998$ & 11066 & $-19 \pm 8(6)$ & $-38 \pm 5(14)$ & 0.61 \\
7 & $16 / 7 / 2006$ & 13933 & $82 \pm 6(2)$ & $82 \pm 9(13)$ & 0.89 \\
\hline
\end{tabular}

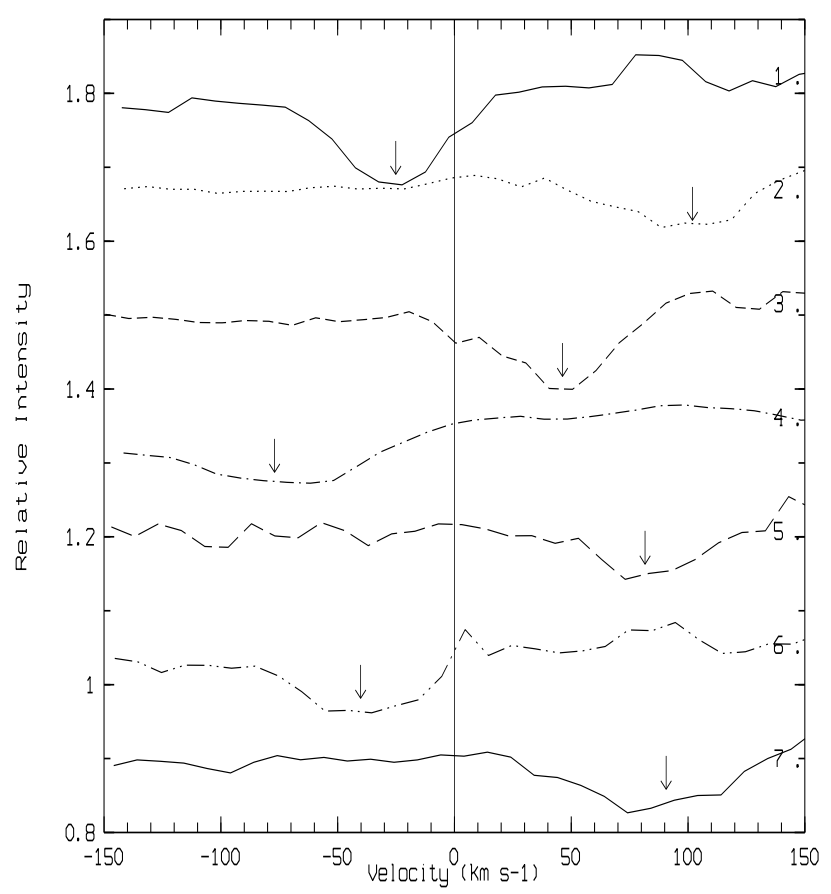

Fig. 1. Spectrum of MWC 314 around the line of SII at $5453.855 \AA$. The spectrograms, normalized to the continuum and vertically shifted, are, from top to bottom 1: ELODIE 28/8/94; 2: ELODIE 7/6/95; 3: ELODIE 16/8/95; 4: AURELIE 5/7/98; 5: AURELIE 21/7/98; 6: ELODIE 9/9/98; 7: ASIAGO 14/7/2006.

and number of lines of these species in parenthesis. The fifth column gives the other (N II, S II, Si II, Ne I) photospheric line radial velocity, standard deviation of the line set in $\mathrm{km} \mathrm{s}^{-1}$, and number of lines of these species in parenthesis. The sixth column gives the proposed phases for the star, which show the photospheric absorption lines (see Sect. 6.1)

We were surprised in finding evidence of drastic photospheric line radial velocity changes with time, while the emission line radial velocity is stable. Their spectral positions indicate radial velocity variations as high as $175 \mathrm{~km} \mathrm{~s}^{-1}$ over approximately 15 days (July 1998). These variations are clearly displayed for the SII absorption line at $5453.855 \AA$ in Fig. 1 . These variations will be discussed in Sect. 6.1.

\section{Analysis of the emission-line fluxes}

\subsection{Line structure}

The emission lines are double-peaked as noticed by Miroshnichenko in 1996. The great majority of the lines are highly blended. In Fig. 2 we show the way we fit each line with four components for the stronger ones, while for the fainter 


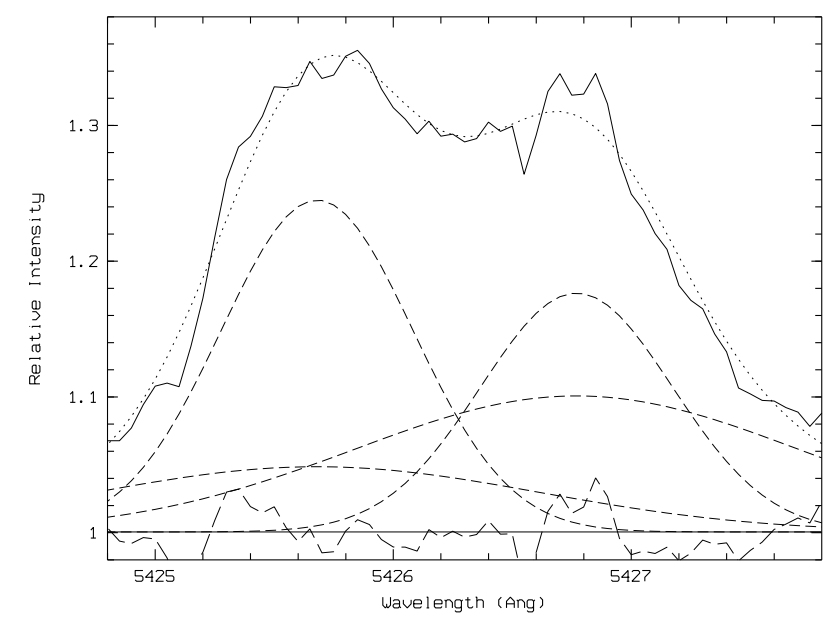

Fig. 2. Emission-line profile of the Fe II line of multiplet 49 at $5425.257 \AA$ observed in 1998 September 9. Ordinate is flux in arbitrary units, abscissa is wavelength at a resolution of 42000 . The observed profile is plotted as a solid line, each line component is represented by a dashed line, the total fit is the dotted line, and the residual is represented by a long dashed curve around the continuum (flux $=1$ ). The $F W H M$ are respectively 0.90 and $2.1 \AA$ (50 and $115 \mathrm{~km} \mathrm{~s}^{-1}$ ) for the narrow and broad components.

Table 3. Mean "blue" and "red" component velocities and standard deviation of the line set of permitted and forbidden Fe II emission lines for the various epochs in $\mathrm{km} \mathrm{s}^{-1}$.

\begin{tabular}{ccccccc}
\hline \hline Date & $\begin{array}{c}\text { Fe II } \\
\text { "blue" }\end{array}$ & $\begin{array}{c}\text { Fe II } \\
\text { "red" }\end{array}$ & $n$ & $\begin{array}{c}{[\mathrm{Fe} \text { II] }} \\
\text { "blue" }\end{array}$ & $\begin{array}{c}{[\mathrm{Fe} \text { II }]} \\
\text { "red" }\end{array}$ & $n$ \\
\hline $28 / 8 / 1994$ & $1 \pm 6$ & $61 \pm 8$ & 101 & $-2 \pm 5$ & $59 \pm 10$ & 13 \\
$7 / 6 / 1995$ & $1 \pm 7$ & $56 \pm 7$ & 125 & $1 \pm 4$ & $55 \pm 7$ & 23 \\
$19 / 8 / 1995$ & $4 \pm 4$ & $64 \pm 6$ & 102 & $4 \pm 7$ & $52 \pm 7$ & 14 \\
$2: 9 / 7 / 1998$ & $4 \pm 6$ & $63 \pm 12$ & 112 & $14 \pm 9$ & $58 \pm 10$ & 24 \\
$21: 22 / 7 / 1998$ & $10 \pm 7$ & $73 \pm 9$ & 39 & $16 \pm 10$ & $67 \pm 9$ & 11 \\
$9 / 9 / 1998$ & $4 \pm 4$ & $61 \pm 5$ & 120 & $7 \pm 6$ & $52 \pm 6$ & 30 \\
$16 / 7 / 2006$ & $0 \pm 9$ & $71 \pm 8$ & 42 & $2 \pm 8$ & $64 \pm 11$ & 8 \\
\hline
\end{tabular}

ones or for heavy blends, only the narrow components were fitted, as the broad ones were too difficult to measure. The line component widths are stable with time.

For the permitted lines of Fe II, as well as Cr II and Ti II, the $F W H M$ are respectively $50 \mathrm{~km} \mathrm{~s}^{-1}$ (velocity broadening $\Delta v=$ $\left.30 \mathrm{~km} \mathrm{~s}^{-1}\right)$ for the narrow components and $115 \mathrm{~km} \mathrm{~s}^{-1}(\Delta v=$ $70 \mathrm{~km} \mathrm{~s}^{-1}$ ) for the broad ones. For the [Fe II] lines, these values are lower, namely, $40 \mathrm{~km} \mathrm{~s}^{-1}\left(\Delta v=25 \mathrm{~km} \mathrm{~s}^{-1}\right)$ for the narrow components and $90 \mathrm{~km} \mathrm{~s}^{-1}\left(\Delta v=60 \mathrm{~km} \mathrm{~s}^{-1}\right)$ for the broad ones of some lines. The FWHM of 2 [Fe III] line $(4658.10,5270.46)$ narrow components are lower than $30 \mathrm{~km} \mathrm{~s}^{-1}\left(\Delta v=20 \mathrm{~km} \mathrm{~s}^{-1}\right)$. The $F W H M$ of the [NII] line 6583.6 (multiplet $1 \mathrm{~F}$ ) is even lower. The narrow component value is $20 \mathrm{~km} \mathrm{~s}^{-1}\left(\Delta v=12 \mathrm{~km} \mathrm{~s}^{-1}\right)$, while a fainter broad component has an FWHM of $44 \mathrm{~km} \mathrm{~s}^{-1}$ $\left(\Delta v=26 \mathrm{~km} \mathrm{~s}^{-1}\right)$.

In Table 3 we give the mean of "blue" and "red" component heliocentric velocities of Fe II and [Fe II] emission lines at each epoch of observation. The difference between the velocities of the "blue" and "red" components of the permitted and forbidden emission lines, and the eventual variations in these differences with time, are smaller than the measurement precision.

In Table 4 the mean values of "blue" and "red" component velocities of $\mathrm{Cr}$ II and Ti II emission lines are displayed.
Table 4. Mean "blue" and "red" component velocities and standard deviation of the line set of $\mathrm{Cr}$ II and Ti II emission lines for the various epochs.

\begin{tabular}{ccccccc}
\hline \hline Date & $\begin{array}{c}\text { Cr II } \\
\text { "blue" }\end{array}$ & $\begin{array}{c}\text { Cr II } \\
\text { "red" }\end{array}$ & $n$ & $\begin{array}{c}\text { Ti II } \\
\text { "blue" }\end{array}$ & $\begin{array}{c}\text { Ti II } \\
\text { "red" }\end{array}$ & $n$ \\
\hline $28 / 8 / 1994$ & $1 \pm 6$ & $57 \pm 13$ & 101 & $0 \pm 5$ & $61 \pm 13$ & 25 \\
$7 / 6 / 1995$ & $0 \pm 5$ & $60 \pm 7$ & 32 & $0 \pm 5$ & $55 \pm 6$ & 43 \\
$19 / 8 / 1995$ & $3 \pm 4$ & $64 \pm 5$ & 26 & $-1 \pm 3$ & $65 \pm 4$ & 26 \\
$2: 9 / 7 / 1998$ & $0 \pm 9$ & $62 \pm 8$ & 29 & $3 \pm 6$ & $60 \pm 10$ & 31 \\
$21: 22 / 7 / 1998$ & $7 \pm 9$ & $70 \pm 8$ & 14 & $5 \pm 8$ & $71 \pm 6$ & 11 \\
$9 / 9 / 1998$ & $5 \pm 6$ & $60 \pm 6$ & 37 & $2 \pm 6$ & $61 \pm 6$ & 45 \\
$16 / 7 / 2006$ & $-4 \pm 23$ & $74 \pm 18$ & 9 & $9 \pm 9$ & $64 \pm 5$ & 7 \\
\hline
\end{tabular}

\subsection{Self-absorption curve}

We recently (Muratorio et al. 2006) studied the environment of the star HD 45677 by means of the self-absorption curve method applied to the numerous metallic emission lines present in the spectra of this object. We adopt here the terminology of this study.

If we analyze the line fluxes of individual $\mathrm{Fe}$ II multiplets in a $\left[\log g f \lambda, \log \frac{F \lambda^{3}}{g f}\right]$ diagram, where the quantity $\frac{F \lambda^{3}}{g f}$ is the normalized flux of an individual emission line and $g f \lambda$ proportional, for a given multiplet, to the optical thickness at the line center (see Viotti et al. 2000), we note that the strongest lines have a normalized flux lower than that of the weaker multiplet lines (see Fig. 3). This indicates that they are affected by a certain amount of self-absorption. The bend of these diagrams, of multiplets with common upper term, helps to determine the relative population of the lower terms of the multiplets by their horizontal shifts with respect to each other by an amount equal to the logarithm of this population ratio (e.g. Kotnik-Karuza et al. 2002). We should similarly be able to derive the mean excitation of the upper terms of multiplets with a common lower term, if the energy range of the lines usually observed were not so small.

If we assume that all the multiplets fit a common theoretical self-absorption curve $Q(\tau)$, we can use the least squares method to determine the parameters $p, X_{\mathrm{c}}, q, Y_{\mathrm{c}}$ needed to overlap the lines of all multiplets to the theoretical curve in an $\left[X-X_{\mathrm{c}}, Y-Y_{\mathrm{c}}\right]$ diagram, where they correspond to the following physical definitions:

$X=\log g f \lambda-p\left(\chi_{1}-\chi_{\mathrm{c}}\right)$

$Y=\log \frac{F \lambda^{3}}{g f}+q\left(\chi_{\mathrm{u}}-\chi_{\mathrm{c}}\right)$

$X_{\mathrm{c}}=1.576-\log \frac{N_{\mathrm{c}}}{g_{\mathrm{c}}}+\log v_{\mathrm{c}}$

$Y_{\mathrm{c}}=\log \frac{S^{\prime}}{d^{2}}+\log \frac{N_{\mathrm{c}}}{g_{\mathrm{c}}}+w-\log \frac{2 \pi \mathrm{e}^{2} h}{m_{\mathrm{e}}}$

and $\log \frac{2 \pi \mathrm{e}^{2} h}{m_{\mathrm{e}}}=16.977$ in cgs units.

The quantities $\chi_{1}$ and $\chi_{\mathrm{u}}$ are the excitation potentials (in $\mathrm{eV}$ ) of the lower and upper levels of each transition, and $\chi_{\mathrm{c}}$ is a characteristic excitation potential taken different from $0 \mathrm{eV}$ in the case of Fe II permitted lines (and equal to $2.75 \mathrm{eV}$ ). The unknown parameters $p$ and $q$ describe the mean population distributions of the lower and upper terms, respectively (Muratorio et al. 1992). In this procedure we assume that the populations of different levels belonging to the same spectroscopic term are approximately 

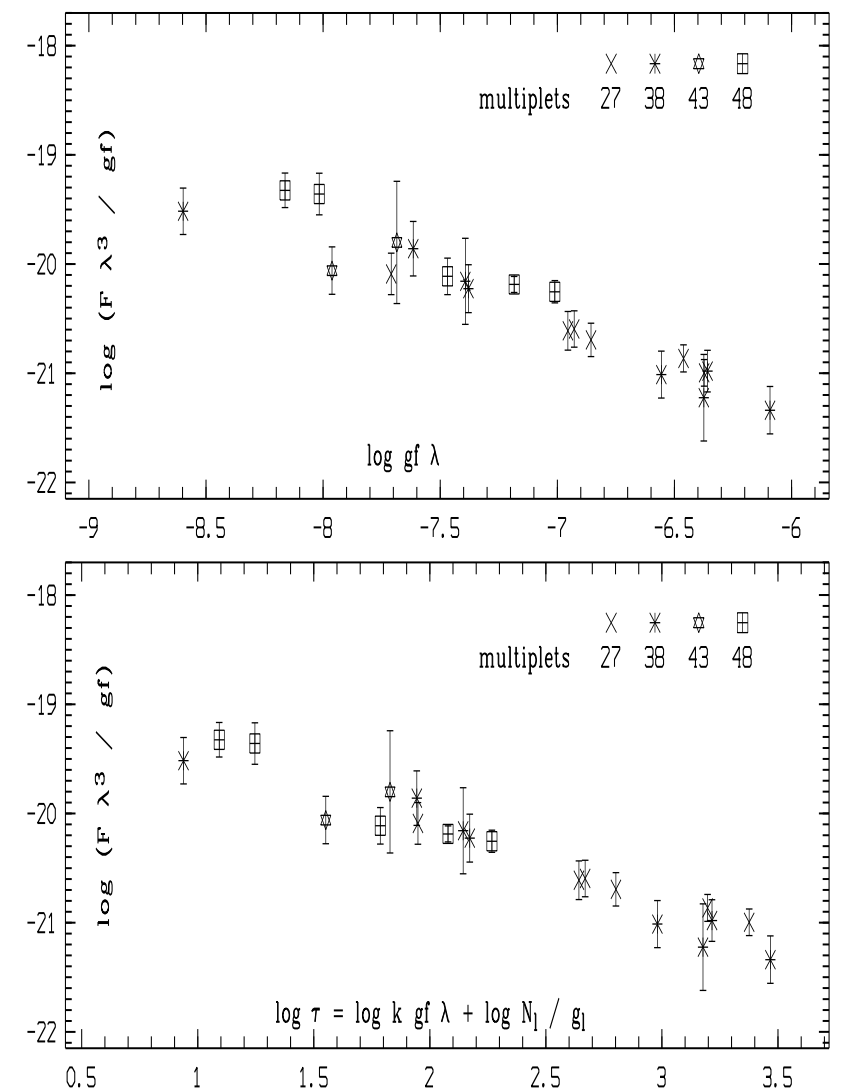

Fig. 3. Upper panel: Log normalized fluxes versus $\log g f \lambda$ plots for the narrow components of Fe II multiplets 27, 38, 43, and 48 having a common upper term $z^{4} \mathrm{D}^{0}$ in the 9/09/1998 spectrum of MWC 314. Lower panel: $\log$ normalized fluxes versus $\log g f \lambda-p \chi_{1}$ plots of multiplets $27,38,43$, and 48 . The points of each multiplet are horizontally shifted by a value corresponding to lower term log population ratios with $p=0.75$.

proportional to their statistical weights, as verified in each multiplet $\left[\log g f \lambda, \log \frac{F \lambda^{3}}{g f}\right]$ diagram, and that all the lower and upper levels follow a Boltzmann-type law with excitation temperatures $T_{l}$ and $T_{\mathrm{u}}$ of 5040/p and 5040/q, respectively. The term $w$ in Eq. (4) describes the overall deviation in the population of the upper (odd) levels of the Fe II transitions with respect to the population of the lower (even) ones. If we assume that the odd and even level population laws link up at an intermediate $\bar{\chi}$, the term turns out to be equal to $w=\left(\bar{\chi}-\chi_{\mathrm{c}}\right)(q-p)$, where for the case of Fe II one can take $\bar{\chi}=4.72 \mathrm{eV}$.

The zero points $X_{\mathrm{c}}$ and $Y_{\mathrm{c}}$ are related to the $\mathrm{Fe}^{+}$column density $\frac{N_{\mathrm{c}}}{g_{\mathrm{c}}}$ at the arbitrarily chosen level $\chi_{\mathrm{c}}$, and to the projected extension of the emitting volume $S^{\prime}\left(S^{\prime}=S \cos i\right)$, through the Eqs. (3) and (4) (Friedjung \& Muratorio 1987; Baratta et al. 1998). The column density $\frac{N_{\mathrm{c}}}{q_{\mathrm{c}}}$ also depends on the parameter $v_{\mathrm{c}}$ (Eq. (3), which is the velocity broadening of the opacity profile. For the theoretical SAC function $Q(\tau)$, we adopted the analytical expression for a Gaussian line profile and a homogeneous emitting envelope given by Friedjung \& Muratorio (1987).

\subsubsection{Narrow Fe II line component analysis}

We first consider the narrow emission components of Fe II in the Aurelie spectrum of the 9/9/1998. For the analysis we adopt Kurucz' (1993) oscillator strengths. To look for self-absorption effects, we analyzed the sum of the "blue" and "red" narrow

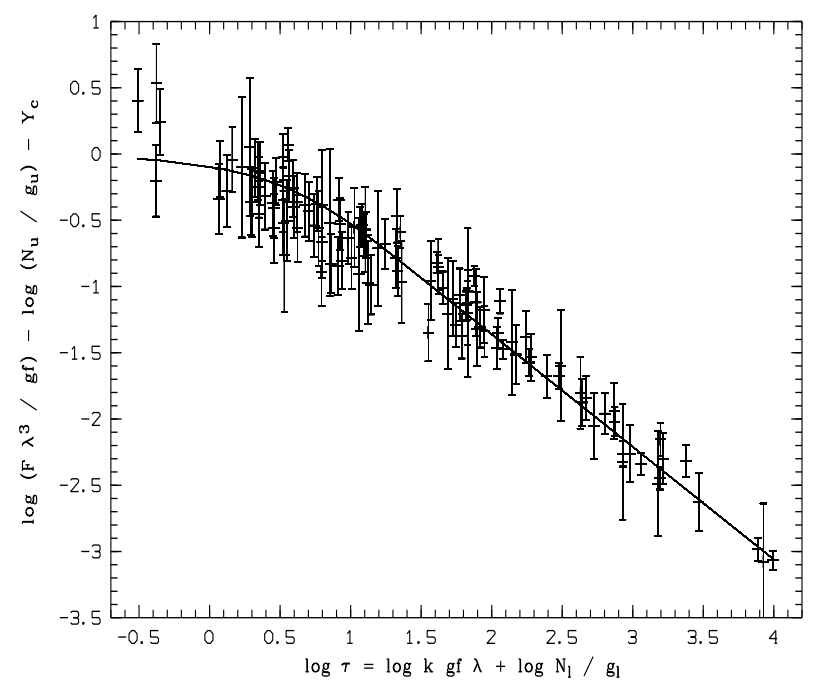

Fig. 4. Global SAC of the Fe II narrow emission components in the 1998 ELODIE spectrum of MWC 314. The representative points are shifted horizontally and vertically by the least squares fitting procedure.The $\log$ population parameters determined correspond to excitation temperatures of $6500 \mathrm{~K}$ for the lower and upper terms.

component line fluxes of individual Fe II multiplets in the $\left[\log g f \lambda, \log \frac{F \lambda^{3}}{g f}\right]$ diagram.

In the upper panel of Fig. 3 we show the diagram for multiplets $27,38,43$, and 48 that have $z^{4} \mathrm{D}^{0}$ as the common upper term. This diagram indicates that most of the lines are affected by a certain amount of self-absorption. The bend in these diagrams allows us to overlap the multiplets in the $\left[\log g f \lambda-p \chi_{l}, \log \frac{F \lambda^{3}}{g f}\right]$ diagram (lower part of Fig. 3), assuming a power law with exponent $-p$. A value of $p=0.75 \pm$ $0.25 \mathrm{eV}^{-1}$ is derived for the exponent of the power law of the lower term population of the multiplets $27,38,43$, and 48 . Similarly, by comparing the 1998 diagrams for multiplets 28 , 37 , and 49 , having $z^{4} \mathrm{~F}^{0}$ as the common upper term, we derive the same mean lower level log population gradient of $p=$ $0.75 \pm 0.25 \mathrm{eV}^{-1}$.

In Fig. 4 we show the result of the least squares fit of the dereddened fluxes of the narrow components of $83 \mathrm{Fe}$ II lines measured in the 1998 ELODIE spectrum of MWC 314. The high excitation $\left(\chi_{\mathrm{u}}>7.5 \mathrm{eV}\right)$ FeII lines are excited similarly to the other permitted lines in MWC 314, which was not the case for HD 45677 (Muratorio et al. 2006). Most of the lines in Fig. 4 lie on the optically thick branch of the SAC. Note that the points are highly dispersed around the suspected bend of the SAC $(\log \tau \simeq$ $0)$. The derived best-fit parameters for the various epochs are summarized in Table 5, where $p\left(\mathrm{eV}^{-1}\right), X_{\mathrm{c}}, q\left(\mathrm{eV}^{-1}\right)$ and $Y_{\mathrm{c}}$ are fitting parameters, and $\chi^{2}$ is the standard deviation. The fit gives a mean lower level population $p$ that is usually rather lower than the mean upper level population parameter $q$.

We adopt for both levels a mean excitation temperature $T_{\text {exc }}$ of $6500_{-1000}^{+1500} \mathrm{~K}$, in fairly good agreement with the values previously found by considering two groups of multiplets having common upper terms.

\subsubsection{Broad Fe II line component analysis}

Because of the difficulty of measuring the broad components of faint Fe II lines, only the broad components of the stronger lines are measured, and consequently all the points lie in the optically 
Table 5. SAC fit parameters for the permitted Fe II narrow component lines.

\begin{tabular}{ccccccc}
\hline \hline Date & $p$ & $X_{\mathrm{c}}$ & $q$ & $Y_{\mathrm{c}}$ & $N$ & red. $\chi^{2}$ \\
\hline $28 / 8 / 94$ & $0.75 \pm 0.11$ & $-9.62 \pm 0.10$ & $0.75 \pm 0.13$ & $-16.54 \pm 0.33$ & 68 & 0.04 \\
$7 / 6 / 95$ & $0.68 \pm 0.08$ & $-9.15 \pm 0.08$ & $0.90 \pm 0.09$ & $-16.48 \pm 0.23$ & 83 & 0.02 \\
$19 / 8 / 95$ & $0.67 \pm 0.10$ & $-9.11 \pm 0.09$ & $0.89 \pm 0.12$ & $-16.60 \pm 0.29$ & 67 & 0.03 \\
$2-9 / 7 / 98$ & $0.75 \pm 0.10$ & $-9.62 \pm 0.14$ & $0.70 \pm 0.11$ & $-16.74 \pm 0.30$ & 80 & 0.05 \\
$21-22 / 7 / 98$ & $0.75 \pm 0.10$ & $-9.44 \pm 0.26$ & $0.95 \pm 0.30$ & $-16.29 \pm 0.79$ & 18 & 0.03 \\
$9 / 9 / 98$ & $0.76 \pm 0.08$ & $-9.62 \pm 0.07$ & $0.79 \pm 0.09$ & $-16.50 \pm 0.26$ & 83 & 0.03 \\
$16 / 7 / 06$ & $0.81 \pm 0.13$ & $-9.30 \pm 0.30$ & $1.04 \pm 0.30$ & $-16.13 \pm 0.83$ & 28 & 0.05 \\
\hline
\end{tabular}

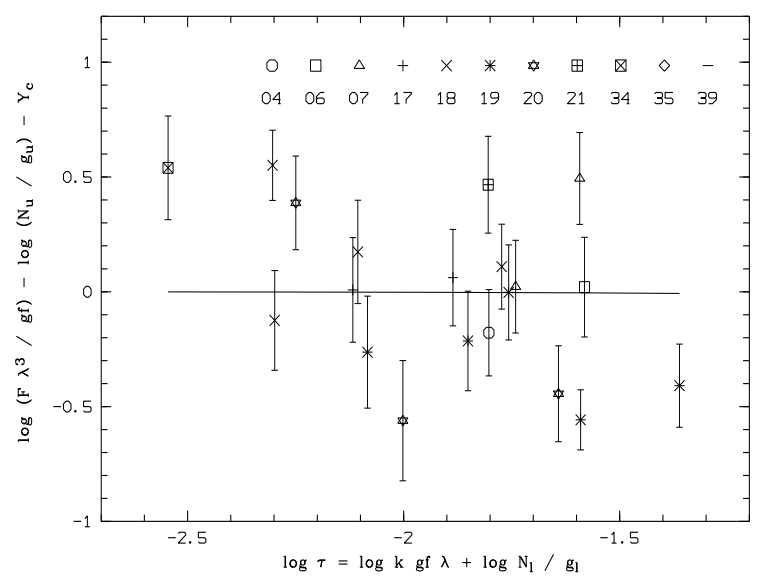

Fig. 5. $\left[X-X_{\mathrm{c}}, Y-Y_{\mathrm{c}}\right]$ diagram for the [Fe II] emission lines of the 1998 AURELIE spectrum. The representative points are shifted horizontally and vertically by $\log$ level population ratios corresponding to excitation temperatures of $10500 \mathrm{~K}$ for the lower and upper terms.

thick part of the $\left[X-X_{\mathrm{c}}, Y-Y_{\mathrm{c}}\right]$ diagram. The excitation temperature determined from the fit of the Fe II emission line narrow components gives a satisfying result for the SAC of the broad components.

\subsection{3. [Fe II] emission lines}

The [Fe II] emission line fluxes are too faint and the line blending too high to permit broad components, if any, to be measured. We adopted the $[\mathrm{Fe}$ II] transition probabilities of Quinet et al. (1996) and took $\chi_{\mathrm{c}}=0 \mathrm{eV}$ for simplicity.

The plot of individual [Fe II] multiplets in the $[\log g f \lambda$, $\left.\log \frac{F \lambda^{3}}{q f}\right]$ diagram shows a faint systematical downward shift of multiplets arising from higher excitation terms, which can be used to determine their upper level population gradient $q$. We have overlapped the [Fe II] multiplets in the $\left[X-X_{\mathrm{c}}, Y-Y_{\mathrm{c}}\right]$ plane in the same way as for permitted $\mathrm{Fe}$ II lines with the least squares method, assuming that the ground and lower $\mathrm{Fe}^{+}$levels have the same excitation temperature (i.e., $p=q=5040 / T_{\mathrm{f}}$ ). The derived SAC diagram shown in Fig. 5 corresponds to a best-fit excitation temperature of the metastable levels $(0-4 \mathrm{eV})$ of $T_{\mathrm{f}}=10500_{-2000}^{+3000} \mathrm{~K}$. These results were obtained from the 28 [Fe II] lines measured on the 1998 ELODIE spectrum. Fewer lines were measured at other epochs, and consequently it is more difficult to get a fit.

Actually, the upper [Fe II] levels are the same as the lower levels of the permitted transitions treated above. Nevertheless, the fact that two such different excitation temperatures have been derived, added to the lower $F W H M$ value of the [Fe II] line narrow components, is strongly suggestive of two different formation regions.

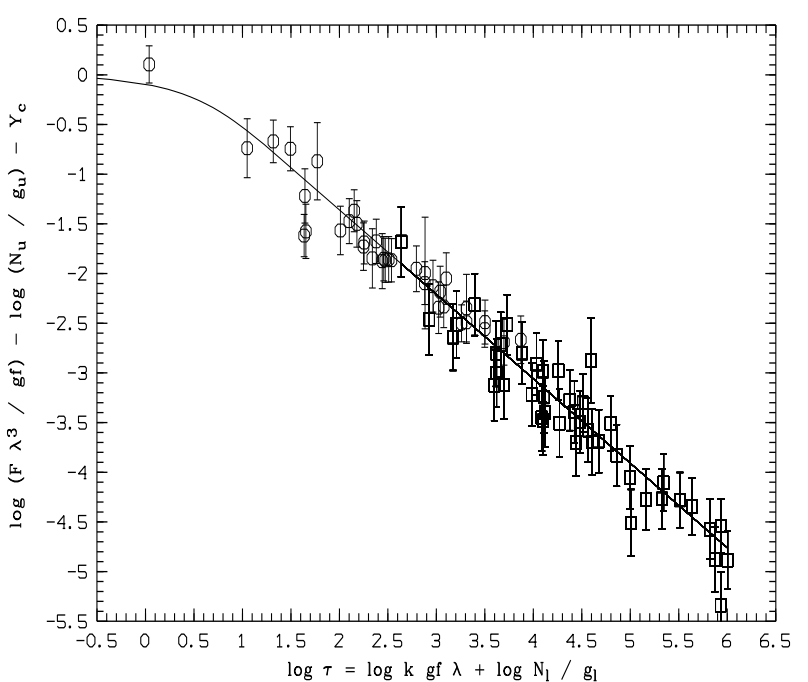

Fig. 6. Global SAC of the Cr II (open circle) and Ti II (open square) narrow emission line components in the 1998 ELODIE spectrum of MWC 314. The representative points are shifted horizontally and vertically by logarithm of the population corresponding to excitation temperatures of $6500 \mathrm{~K}$ for the lower and upper terms.

\subsubsection{Cr II and Ti II line SAC analysis}

The 36 lines of $\mathrm{Cr}$ II lead to an SAC diagram, using again, for both the lower and upper levels, the excitation temperature we derived from the lower levels of the narrow Fe II emission line components at the same date. In Fig. 6 the SAC of the Cr II and the Ti II narrow emission components in the ELODIE 1998 spectrum is displayed. In this diagram all the $\mathrm{Cr}$ II line component representative points, except one, lie in the optically thick part of the SAC.

The 44 lines of Ti II lie in the optically thick part of the $\left[X-X_{\mathrm{c}}, Y-Y_{\mathrm{c}}\right]$ diagram (Fig. 6).

\section{Line profiles}

Though Zickgraf (2003) found that it was not necessary to invoke rotation to produce double-peaked profiles, if we account for the superposition of lines in blends, we find that the central part of the profiles can be understood as being usually not far from the symmetrical profiles produced by a rotating disk, without the clear signs of asymmetry produced by expansion or contraction near a photosphere. This, together with the different widths of forbidden and permitted lines (Sect. 4.1), leads us to think that a rotating disk might be present in MWC 314.

A rotating accretion disk was proposed by Bensammar et al. (1983) for the Magellanic Cloud B[e] supergiant S22, and then Zickgraf et al. (1986) proposed an outflowing disk model for all B[e] supergiants. MWC 314 (Miroshnichenko 1996), 
B[e] supergiants in general (Zickgraf et al. 1986), as well as LBVs are understandable as evolved stars. However the situation concerning a possible evolutionary link between $\mathrm{B}[\mathrm{e}] \mathrm{su}-$ pergiants and LBVs is not clear (Zickgraf 2006). The presence of what is probably a Keplerian disk was also supposed by Muratorio et al. (2006) for the much less luminous B[e] HD 45677, whose properties lead specialists to suppose that it is young, unlike $\mathrm{B}[\mathrm{e}]$ supergiants, so the disk may be an accretion disk. In the case of classical Be stars, two peaks can be seen for inclinations greater than $15-18^{\circ}$ at a resolution of 15000 $\left(20 \mathrm{~km} \mathrm{~s}^{-1}\right)$. They are seen in Fe II emission line profiles in the spectra of classical Be stars, even when not seen in the Balmer line profiles (Zorec 2007).

It is reasonable to suppose that the disk of MWC 314 is produced by a very low-velocity outflow as for B[e] supergiants in general rather than by accretion, unlike in the cases of various sorts of interacting binaries such as cataclysmic binaries, but the indications of binarity of MWC 314, discussed in Sect. 6.1, lead us not to completely discard the accretion disk explanation. When a disk contains ejected material possessing angular momentum, the conservation of angular momentum leads to a velocity of rotation that, if $r$ is the radial distance from the center, varies as $r^{-1}$, while for a Keplerian disk it varies as $r^{-0.5}$. Conservation of angular momentum is physically more plausible, but the question of whether such disks are or are not Keplerian is unresolved (Lamers 2006).

The profile wings, also seen in the spectra of HD 45677, were explained by Muratorio et al. (2006) by an outflow or inflow. In the case of MWC 314, we do observe wings in the Fe II, $\mathrm{Cr}$ II, and Ti II permitted line profiles (see Sect. 4.1). It is hard to measure broad components in the [Fe II] line profiles.

The central parts of the profiles of the forbidden lines, which can be expected to be formed farther from the star with a lower density below the critical density of $2 \times 10^{8} \mathrm{~cm}^{-3}$ for the $7155 \AA$ line (Zickgraf 2003), are narrower than those of permitted lines, as expected for models having a Keplerian disk or a rotating disk with conservation of angular momentum. The (asymmetric) $[\mathrm{N} \mathrm{II}] 6583 \AA$ line with an even lower critical density of $9 \times$ $10^{4} \mathrm{~cm}^{-3}$ (Zickgraf 2003) appears to be even narrower, as is also the case for the (asymmetric) [Fe III] 4658 and $5270 \AA$ A lines. All these observational data are consistent with the presence of a rotating disk.

The profiles displayed in Figs. 7 for Fe II permitted lines, and 8 for $[\mathrm{Fe} \mathrm{II}]$ lines are the histograms of the radial velocities of the emitting material of optically thin disks superposed on the profiles observed with ELODIE on the 1998 September 9. The disks used for these models are in each case Keplerian or have conservation of angular momentum. A small inflow or outflow of emitting material is supposed to be present perpendicular to each face of the disk in order to obtain broad components similar to the one observed for the Fe II lines. The amount of this material is a free parameter for the model.

To obtain line profiles similar to the Fe II lines observed in MWC 314, we have to suppose that the disks are inclined $25^{\circ} \pm$ $5^{\circ}$ to the plane of the sky. The velocity at the star's photospheric radius $(r=1)$ is taken equal to $340 \pm 50 \mathrm{~km} \mathrm{~s}^{-1}$ in the Keplerian hypothesis, and to $300 \pm 50 \mathrm{~km} \mathrm{~s}^{-1}$ in the conservation of angular momentum case. In the first hypothesis, Fe II emission occurs from $2 \pm 1$ times up to $15 \pm 2$ times the photospheric radius. Profiles similar to the one observed for forbidden [Fe II] lines of MWC $314\left(40 \mathrm{~km} \mathrm{~s}^{-1}\right)$ are then emitted in the same disk from distances to the star between $12 \pm 2$ to $15 \pm 2$ star radii. In the second hypothesis, Fe II emission occurs from $1.5 \pm 0.3$ times

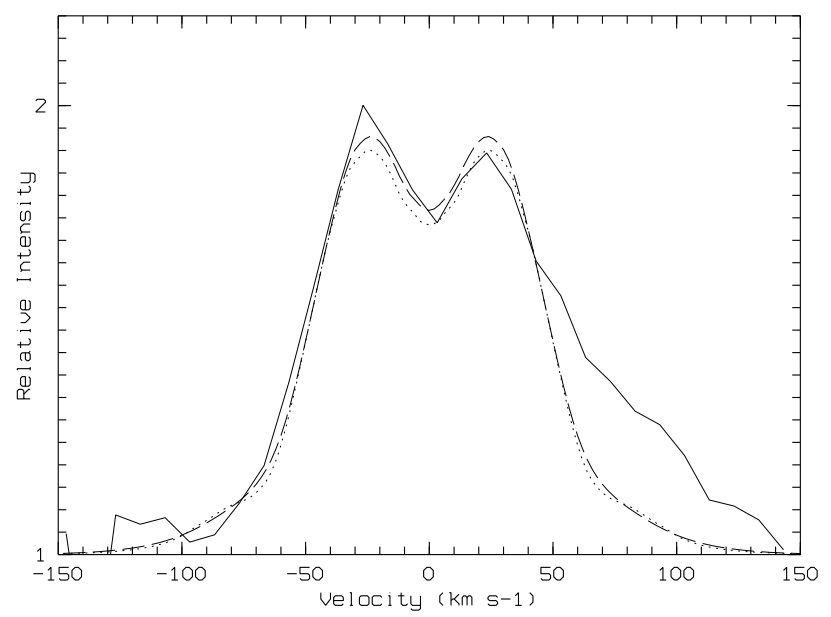

Fig. 7. Histograms of the emitting material radial velocities of an optically thin disk inclined $25^{\circ}$ on the sky plane superposed on the Fe II 4629.339 line observed the $9 / 9 / 1998$ (the line is blended on the red side with the 4630 N II and Mg II lines).The Keplerian disk model profile is plotted as a dotted line. The disk rotating with conservation of angular momentum model profile is plotted as a long dashed line.

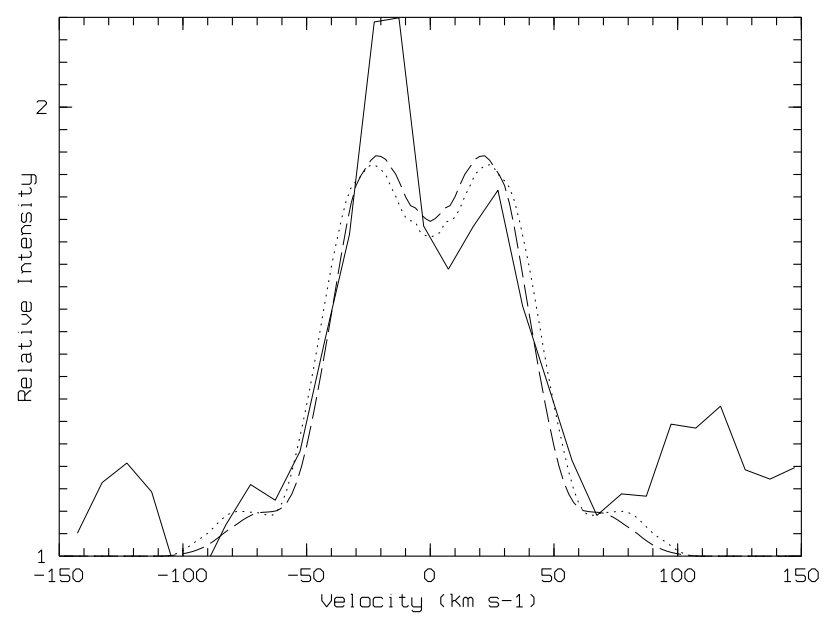

Fig. 8. Histograms of the emitting material radial velocities of an optically thin disk inclined $25^{\circ}$ on the sky plane superposed on the [Fe II] 5158.810 line observed the 9/9/1998 (the line is blended on the blue side with the [Fe II] 5158.000 line, which, although fainter, at least partly explains the assymetric profile). The Keplerian disk model profile is plotted as a dotted line. The disk rotating with conservation of angular momentum model profile is plotted as a long dashed line.

the star photosphere up to $4 \pm 0.3$ times this radius, while [Fe II] is emitted from a distance to the star between $3 \pm 0.3$ to $4 \pm$ 0.3 times this radius.

\section{Discussion}

\subsection{Variations in absorption-line radial velocities}

The variations in absorption-line radial velocities could be caused by binary motion or by some sort of pulsation. It is possible to examine these possibilities in more detail.

The effective temperature and luminosity were determined by Miroshnichenko (1996) as $30000 \mathrm{~K}$ and for $\log \left(\frac{L}{L \odot}\right)=6.2$, leading to a radius of $50 R_{\odot}$. Comparison with evolutionary tracks, including mass loss, led to an initial mass on the order of $80 R_{\odot}$. Miroshnichenko et al. (1998) revised these estimates to obtain an effective temperature of about $25000 \mathrm{~K}, a \log \left(\frac{L}{L \odot}\right)$ 


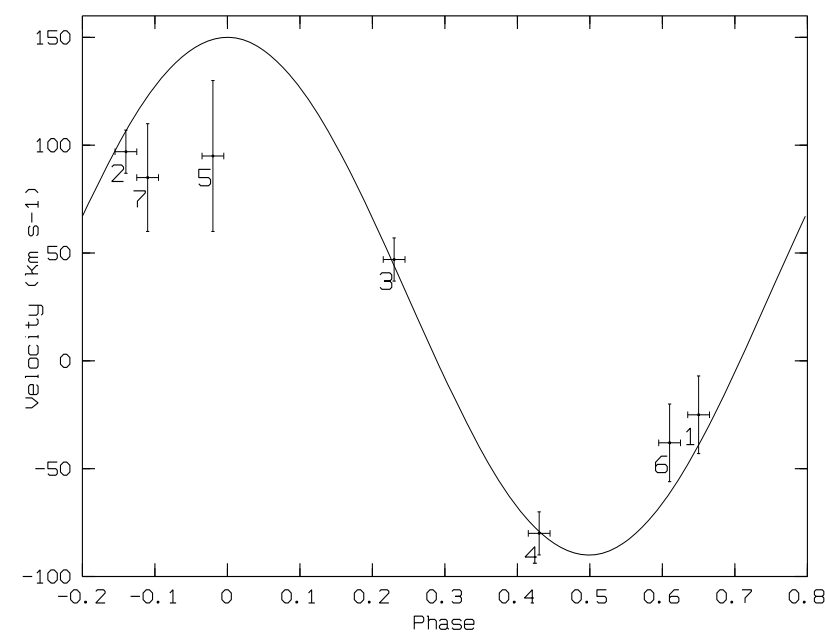

Fig. 9. Phases in the rotation for a $30.7 \pm 0.5$ days period. The different phase velocity values are labeled with the first column number of each corresponding line in Table 2 (1: 28/8/1994; $2: 7 / 6 / 1995 ; 3: 19 / 8 / 1995$; 4: 2-9/7/1995; 5: 21-22/7/1998; 6: 9/9/1998; 7: 16/7/2006).

of 6.1 , and a radius of $60 R_{\odot}$. It may be noted that such estimates place MWC 314 close to the Humphreys-Davidson limit to stellar luminosity in the temperature luminosity diagram. The estimates of the second of these papers, with a mass of $60 M_{\odot}$ at the present time after mass loss, can be used to examine the binary explanation. Unfortunately the observations are sparse and badly spaced in time. The most rapid absorption line radial velocity change of Table 2 is not far from $200 \mathrm{~km} \mathrm{~s}^{-1}$ for 16 days in July 1998 from spectra taken with the same AURELIE spectrograph. Assuming a nearly circular orbit, which suggests a maximum period on the order of 30 days, the radial velocity variation implies a minimum orbital velocity of $100 / \sin i_{\mathrm{o}} \mathrm{km} \mathrm{s}^{-1}$, where $i_{\mathrm{o}}$ is the inclination of the orbit of the companion. The mass function of what according to the binary explanation must be a single-lined spectroscopic binary is for these parameters equal to $3.1 M_{\odot}$. This is consistent with reasonable values for the separation between the stellar components of the binary and the inclination. Such results, though preliminary, do not at least suggest any basic contradiction in the binary explanation.

Our data are consistent with an orbital period of $30.7 \pm$ 0.5 days, which satisfy the observed radial velocities in Table 2 , for an orbital velocity of $120 \mathrm{~km} \mathrm{~s}^{-1}$, as can be seen in Fig. 9 . It should be noted that Miroshnichenko (1996) presented initial analysis of a $B$-band photometric dataset and found evidence of a 4.16 day period (see their Sect. 2.1, Fig. 3).

The variations in absorption-line radial velocity may perhaps be explained in another way. The nature of variations in LBVs is uncertain; they have been attributed to "strange" modes of pulsation. A short review is presented by Glatzel \& Chernigovski (2001). Such modes seem in general not to be periodic, but to be rather chaotic for massive stars, with velocities on the order of $100 \mathrm{~km} \mathrm{~s}^{-1}$. A disturbance with a constant velocity of this order would moreover take about $4 \times 10^{5} \mathrm{~s}$ or a few days to cross a distance equal to the radius of MWC 314.

\subsection{Column density}

From the position of the bend in the SAC plots of Fe II lines (Fig. 4), we are able to determine the minimum value for $X_{\mathrm{c}}$ and hence the minimum value of the $\mathrm{Fe}^{+}$column density of this region.
We have to estimate the velocity broadening of the opacity profile $v_{\mathrm{c}}$. The narrow components of the emission lines of MWC 314 should be formed in an equatorial disk seen inclined $25^{\circ}$ to the sky plane (Sect. 5). As we are observing this disk in rotation without being in its plane, the line broadening along a line of sight is a function of the thermal broadening velocity of $\mathrm{Fe}^{+}$. The local velocity dispersion for a mean temperature of $6 \times$ $500 \mathrm{~K}$ is given by $v_{\text {th }}=0.215 \times 10^{5} \sqrt{T / \mu}$, where $T$ is the temperature and $\mu=55.85$ for $\mathrm{Fe}^{+}$(Baratta et al. 1998) is the atomic weight, and consequently, $v_{\mathrm{c}}=2 * v_{\text {th }}=4.6 \mathrm{~km} \mathrm{~s}^{-1}$.

The value of the column density at $\chi_{\mathrm{c}}=2.75 \mathrm{eV}$ is $7 \times 10^{16}$ $\left(\log \left(\frac{N_{\mathrm{c}}}{g_{\mathrm{c}}}\right)=16.86 \pm 0.25\right)$. If we extrapolate this column density to $0 \mathrm{eV}$ using 0.75 as the exponent of the power law of the lower term's population, we obtain a value of $9.1 \times 10^{18}$ in $\operatorname{cgs}(4.5 \times$ $\left.10^{18}<\log \left(\frac{N_{0}}{g_{0}}\right)<1.8 \times 10^{19}\right)$.

We assumed the same Boltzmann population distribution for all metastable levels. One can expect LTE at least for those having a lower excitation potential. The calculations of Verner et al. (2002) suggest that Boltzmann's law is obeyed up to $2.3 \mathrm{eV}$, with deviations as functions of excitation potential for higher metastable levels. Therefore, if we are very conservative and suppose that the mean excitation temperature down to $0 \mathrm{eV}$ is $10000 \mathrm{~K}$, typical of $\mathrm{H}$ II regions, as well as being close to what we find here for the less excited forbidden [Fe II] lines with lower levels at or near $0 \mathrm{eV}$, the column density values might be lower by a factor of about 1.4 dex.

In the case of the forbidden lines, the vertical overlapping of the multiplets provides the zero point of the ordinates: $Y_{\mathrm{f}}=-13.6 \pm 0.1$. This quantity is related to the total number $\bar{N}\left(\mathrm{Fe}^{+}\right)$of $\mathrm{Fe}^{+}$ions in the [Fe II] emitting volume through the relationship (see Baratta et al. 1998) $\log \bar{N}\left(\mathrm{Fe}^{+}\right) / d^{2}=$ $Y_{\mathrm{f}}+\log U+16.977$, where $U$ is the partition function of $\mathrm{Fe}^{+}$. Assuming for MWC 314 a distance of $3.0 \pm 0.2 \mathrm{kpc}$ (Miroshnichenko 1996), we obtain $\log \bar{N}\left(\mathrm{Fe}^{+}\right)=49.1 \pm 0.4$ which in 1998 gives, for a cosmic abundance, a total hydrogen mass of $4.8 \times 10^{29} \mathrm{~g} \pm 0.4$ dex.

Notice that the minimum $\log \bar{N}\left(\mathrm{Fe}^{+}\right)=48.2 \pm 0.8$ value in the permitted $\mathrm{Fe}$ II emission region, corresponding to the minimum S' of Sect. 6.3 below, is close to the forbidden one.

\subsection{Size of the emitting region}

From the fit results summarised in Table 5 we can calculate $S^{\prime}$ the surface area perpendicular to the line of sight of the Fe II line emitting region.

We obtain for the typical radius of the narrow line emitting region $\log R=13.55 \pm 0.25\left(\log S^{\prime}=27.6 \pm 0.5\right)$ if $w=0$ (Eq. (4)). We expect the odd terms to be largly underpopulated with respect to the even terms (see Fig. 13 in Verner et al. 2002), making $w$ in fact small. Examination of Table 5 suggests that $p$ is somewhat less than $q$ perhaps by about 0.2 . As a result, the minimum values of $S^{\prime}$ and $R$ may be slightly too high; the real minimum value of $\log R$ will then be about 0.2 less than for $w=0$.

The radius of MWC 314 was estimated to be close to $60 R_{\odot}$ $\left(4.2 \times 10^{12} \mathrm{~cm}\right)$ (Miroshnichenko 1996). According to the models we presented in Sect. 5, the Fe II permitted lines should be emitted from a region whose surface area perpendicular to the line of sight is that of a disk of 15 stellar radii $\left(\log S^{\prime}=28.1\right)$ in the Keplerian disk case, and 4 stellar radii $\left(\log S^{\prime}=26.9\right)$ in the angular momentum conservation case. These values are inside or 
not far from the limits of our determination by the SAC method. All the quantities are in cgs units.

\section{Conclusions}

We confirm the binarity for MWC 314 suspected by Wisniewski et al. (2006). The periodicity needs nevertheless to be improved with a higher sampling frequency. The $\mathrm{Cr}$ II, Ti II, and Fe II emission lines have a complex structure. They are doublepeaked, with each of these two $60 \mathrm{~km} \mathrm{~s}^{-1}$ separated components, composed of a narrow $\left(\Delta v=30 \mathrm{~km} \mathrm{~s}^{-1}\right)$ and a broad $\left(\Delta v=70 \mathrm{~km} \mathrm{~s}^{-1}\right)$ component. At the same time, the Fe II forbidden line components are narrower namely, $\Delta v=25 \mathrm{~km} \mathrm{~s}^{-1}$. The broad components, if present, cannot be observed.

We derived physical parameters of the line-emitting region. The permitted-line, mean excitation temperature is found to be $T_{\text {exc }}=6500_{-1000}^{+1500} \mathrm{~K}$ for both the lower and the upper levels of $\mathrm{Fe}$ II transitions. The typical minimum radius of this line-emitting region is $3.5 \times 10^{13} \mathrm{~cm}\left(2.0 \times 10^{13} \mathrm{~cm}<R<6.3 \times 10^{13} \mathrm{~cm}\right)$. The forbidden-line excitation temperature of the upper levels is $T_{\text {exc }}=10500_{-2000}^{+3000} \mathrm{~K}$. Our results are consistent with line formation in a rotating disk around a star. The very simple model we introduced in our study of HD 45677 gives satisfying results for MWC 314 too. We cannot tell wether this disk is Keplerian or a type of angular momentum conservation. For a disk inclined $25 \pm 5^{\circ}$ on the plane of the sky, we obtained line profiles similar to the observed ones, both for the permitted and forbidden $\mathrm{Fe}$ II lines. In the framework of these very simplified geometrical model, we argue that the forbidden Fe II lines are emitted farther out in the disk than the permitted Cr II, Ti II, and Fe II lines. The sizes of the emitting region deduced from these models agree with the one we derived from the fit of the normalized line fluxes.
Acknowledgements. The authors are very grateful to $\mathrm{H}$. Zhang for providing physical data on [Fe III] lines, to J. Zorec for information on Be stars, and to the referee for his valuable comments.

\section{References}

Baratta, G. B., Friedjung, M., Muratorio, G., Rossi, C., \& Viotti, R. 1998, The Self Absorption Curve Method, A Users ' Munual, IAS Internal Report, December 1998

Bensammar, S., Friedjung, M., Muratorio, G., \& Viotti, R. 1983, A\&A, 126, 427

Friedjung, M., \& Muratorio, G. 1987, A\&A, 188, 100

Glatzel, W., \& Chernigovski, S. 2001, in P Cygni 2000; 400 Years of Progress, ASP Conf. Ser., 233

Kotnik-Karuza, D., Friedjung, M., \& Selvelli, P. L. 2002, A\&A, 381, 507

Kurucz, R. L. 1993, SAO KURUCZ CD-ROM, 18

Lamers, H. G. J. M. L. 2006, in Stars with the B[e] Phenomenon, ed. M. Kraus, \& A. S. Miroshnichenko, ASP Conf. Ser., 355, 371

Marston, A. P., \& McCollum, B. 2008, A\&A, 477, 193

Merill, P. W. 1927, ApJ, 65, 286

Miroshnichenko, A. S. 1996, A\&A, 312, 941

Miroshnichenko, A. S., Fremat, Y., Houziaux, L., et al. 1998, A\&AS, 131, 469

Muratorio, G., Viotti, R., Friedjung, M., Baratta, G. B., \& Rossi, C. 1992, A\&A, 258,423

Muratorio, G., Rossi, C., \& Friedjung, M. 2006, A\&A, 450, 593

Quinet, P., Le Dourneuf, M., \& Zeippen, C. J. 1996, A\&AS, 120, 361

Swensson, J. W. 1942, ApJ, 97, 226

Verner, E. M., Gull, T. R., Bruhweiler, F., et al. 2002, ApJ, 581, 1154

Viotti, R. F., Spector, N., Baratta, G. B., \& Rossi, C. 2000, A\&A, 363, 343

Wisniewski, J. P., Babler, B. L., Bjorkman, K. S., et al. 2006, PASP, 118, 820

Zickgraf, F. J. 2003, A\&A, 408, 257

Zickgraf, F.-J. 2006, in Stars with the B[e] Phenomenon, ed. M. Kraus, \& A. S. Miroshnichenko, ASP Conf. Ser., 355, 211

Zickgraf, F.-J., Wolf, B., Stahl, O., Leitherer, C., \& Appenzeller, I. 1986, A\&A, 163,119

Zorec, J. 2007, Private communication 